\title{
Comments: "TEP versus Lichtenstein, Which One to Choose? A Retrospective Cohort Study"
}

Mehmet Fatih Can ${ }^{1}$

1. Professor of General Surgery University Of Lokman Hekim, Çankaya/Ankara, Turquia. Contact: mfcanmd@gmail.com

Minimally invasive approaches are the trending topic concerning surgery. They provide better postoperative patient satisfaction and lower hospitalization duration, resulting in lower costs. There are now minimally invasive approaches even for major surgeries such as hepatectomies and colectomies, so it is the time to revise the guidelines for inguinal hernioplasty surgical approaches. There is no doubt regarding the superiority of tension-free techniques for inguinal hernioplasty, but with the development of new surgical technologies, laparoscopic tension-free hernioplasties are the main rivals of conventional open tension-free hernioplasties.

The article "TEP versus Lichtenstein, Which One to Choose? A Retrospective Cohort Study" weighs in on laparoscopic inguinal hernioplasties by comparing it with conventional techniques1. This article tries to fill the gap in the literature around the superiority of laparoscopic inguinal hernioplasty. Future studies stemming from this one are likely to give surgeons enough confidence to update the current guidelines, which some have already started to change. Thus, I hope clinicians who read this article will highly benefit from the new perspective it presents to readers.

\section{REFERENCE}

1. Urkan M, Subutay PY. TEP versus Lichtenstein, which one to choose? A retrospective cohort study. Rev Assoc Med Bras 2019; 65(9)1201-1207 